Doh Jeing Yong, MBBS, MRCS, DOHNS' Abd Majid Md Nasir, MBBS, MS (ORL-HNS)' Bee See Goh, MBBS, MS (ORL-HNS)²

'Department of Otorhinolaryngology Hospital Kuala Lumpur, Malaysia

${ }^{2}$ Department of Otorhinolaryngology Universiti Kebangsaan Malaysia Medical Centre
Correspondence: Dr. Doh Jeing Yong Department of Otorhinolaryngology, Hospital Kuala Lumpur, Jalan Pahang, 50586 Kuala Lumpur, Malaysia

Phone: (+601) 22719921

Fax: (+603) 26916725

Email drdjyong@gmail.com

Reprints will not be available from the authors.

The authors declared that this represents original material that is not being considered for publication or has not been published or accepted for publication elsewhere, in full or in part, in print or electronic media; that the manuscript has been read and approved by the authors, that the requirements for authorship have been met by the authors, and that the authors believe that the manuscript represents honest work.

Disclosures: The authors signed a disclosure that there are no financial or other (including personal) relationships, intellectual passion, political or religious beliefs, and institutional affiliations that might lead to a conflict of interest.

\section{Middle Ear Carcinoma Masquerading as an Aural Polyp}

\begin{abstract}
Objective: To present a case of middle ear carcinoma masquerading as an aural polyp and describe our experience with the clinical presentation, management and outcome of an elderly patient with this pathology.
\end{abstract}

\section{Methods:}

\author{
Design: Case Report \\ Setting: Tertiary Public Hospital \\ Patient: One
}

Results: A 63-year-old female presented with an aural polyp and preceding symptoms of inner ear disturbances followed by otorrhea and otalgia. CT scans revealed an erosive lesion occupying the entire middle ear cleft, external ear canal and mastoid cavity with involvement of inner structures. A repeat biopsy subsequently revealed malignancy leading to a diagnosis of middle ear carcinoma. The patient was offered surgical treatment but opted for radiotherapy and subsequently defaulted follow-up.

Conclusion: Middle ear carcinoma is rare and can masquerade as a benign aural polyp. Symptoms of severe otalgia and inner ear disturbances are indicators of possible malignancy, as are recentonset symptoms of otitis media developing over a relatively short course later in life. A high index of suspicion is needed to avoid late diagnosis. Repeat deeper aural tissue biopsy is needed to exclude malignancy. Computed tomography imaging is indispensable in delineating tumor extent and aids in tumor staging as well as prognostication. Surgical resection with clear tumor margins, followed by postoperative radiotherapy, is the preferred choice of treatment. Sole radiotherapy is reserved for tumors of small volume as well as in cases where surgery is not feasible.

\section{Keywords: middle ear carcinoma; aural polyp; temporal bone carcinoma}

Carcinomas of the middle ear are rare, with occurrences of less than $0.2 \%$ of head and neck tumors. ${ }^{1}$ The presenting symptoms and signs share similarities to those of chronic ear infection as well as cholesteatoma. Therefore, early diagnosis is rarely possible resulting in late-stage disease on presentation. No gender predilection is noted and they are usually reported to occur in patients with histories of irradiation as well as long-standing ear infection. ${ }^{2}$ We report the case of a patient diagnosed with middle ear carcinoma masquerading as aural polyp. 


\section{CASE REPORT}

A 63-year-old female with no known medical illness presented with gradual onset, right non-pulsatile tinnitus, hearing loss and episodic vertigo for eight months. Subsequently, she experienced persistent right otalgia associated with intermittent mucoid otorrhea and recently felt a mass at the meatus of the right ear canal. (Figure 1) There was no headache or facial weakness. On examination, the right pinna was normal and non-tender. No mastoid swelling was noted. The external meatus was occupied with a greyish mass extending through the entire right ear canal. The mass was firm on probing and not pulsatile. The tympanic membrane was not visualized. Regional lymph nodes were not palpable. Tuning fork tests revealed right conductive hearing loss. Facial nerve examination was normal. Nystagmus and cerebellar signs were absent. The intraoral, indirect laryngeal and post nasal mirror examinations were normal. No neurological deficits were noted. With an initial impression of aural polyp, an aural biopsy was taken and the histopathological assessment reported as infarcted polyps with no evidence of malignancy. Nonetheless, the patient was referred to our centre for further management. Pure tone audiometry showed significant right conductive hearing loss with an average air-bone gap of $35 \mathrm{dbHL}$. (Figure 2) Computed tomography of the temporal bone showed evidence of an extensive soft tissue mass occupying the right external ear canal, middle ear and mastoid cavity with erosion of the bony external ear canal, ossicles and lateral semicircular canal. (Figure 3) The patient underwent re-biopsy of the right ear canal mass under general anesthesia and the histopathological assessment reported findings of squamous cell carcinoma. A final diagnosis of temporal bone squamous cell carcinoma T4NOMO was made. However, the patient was not keen for surgery and radiotherapy was planned. Unfortunately, the patient defaulted subsequent follow up.

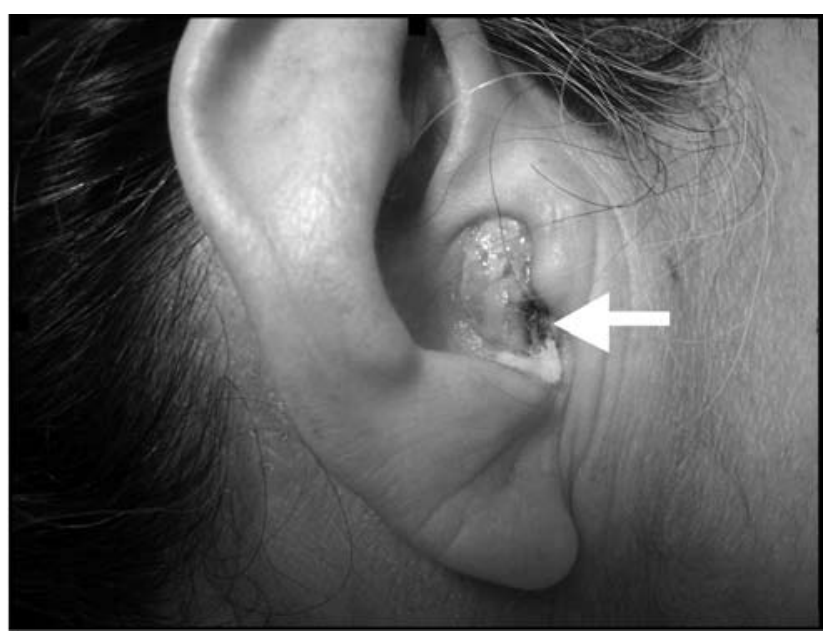

Figure 1. Right aural mass occupying the entire ear canal and external meatus (white arrow).

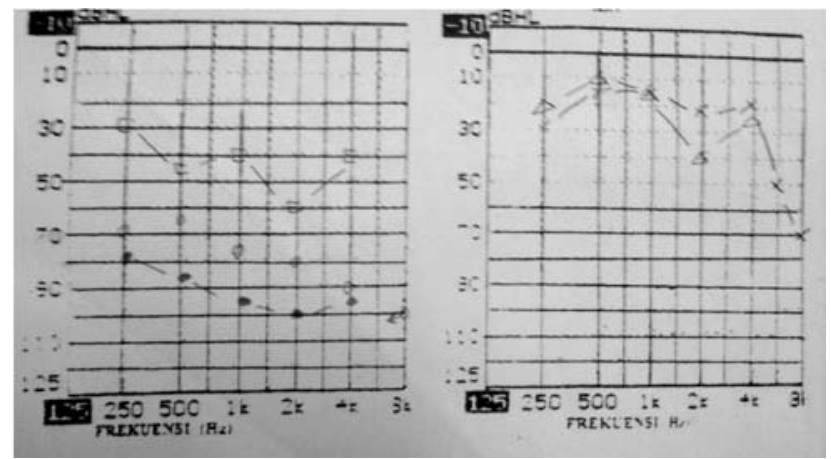

Figure 2. The pure tone audiogram threshold showing mixed hearing loss in the right ear.

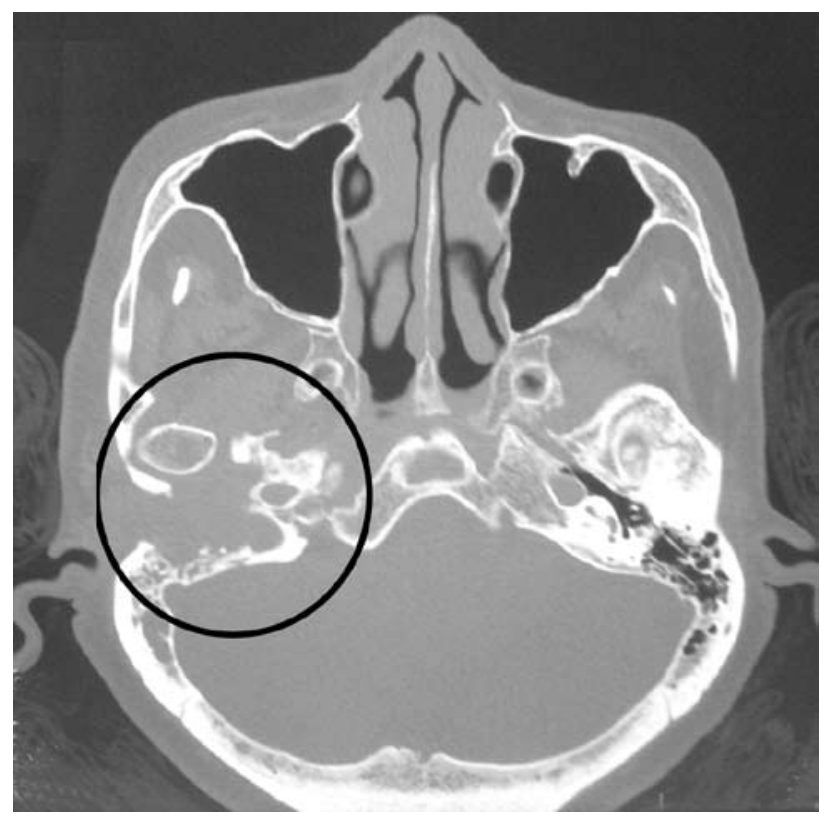

Figure 3. Computed tomography of the temporal bone axial view showing a soft tissue mass occupying the entire middle ear and mastoid cavity (black ring). Inner ear bony erosions are prominent with loss of middle ear and inner ear structures.

\section{DISCUSSION}

Malignancies of the temporal bone are rare, accounting for less than $0.2 \%$ of head and neck tumors.' These occurrences include cancer arising from the skin of the pinna that extend into the temporal bone, metastatic temporal bone lesions as well as primary tumors of the external ear canal, middle ear, mastoid cavity, petrous apex. Nonetheless, the literature reviews on primary temporal bone carcinoma of middle ear origin are few.

Primary temporal bone carcinoma is often diagnosed late as the presenting symptoms share similarities with chronic ear infections. Otorrhea, otalgia and bloody otorrhea were among the commonest presentations. ${ }^{2}$ Deafness, tinnitus, vertigo, facial nerve palsy as well as referred pain of upper teeth have been reported. ${ }^{3,45}$ Therefore, middle ear carcinoma should be considered when refractory aural polyp, severe otalgia and long-standing history of otorrhea are observed. Absence of 
history of otorrhea denotes possible external ear origin. ${ }^{6}$ Furthermore, there are frequent associations with chronic otitis media and aural polypectomy procedure.? Other risk factors include previous history of irradiation as well as human papilloma virus infection.

This case report highlights the atypical presentation of middle ear carcinoma masquerading as an infarcted polyp. Chronologically, our patient initially presented with inner ear symptoms for eight months followed by recent otorrhea and aural polyp. Because the onset of otorrhea in an elderly lady was only for one month duration prior to the notice of an aural mass, this should have raised a red flag for earlier intervention as the long-standing symptoms of tubotympanic otitis media usually begin in childhood. The rather atypical sequence of symptoms in this case explains the tumor origin and extensive involvement of the inner ear at time of diagnosis. Therefore, we feel that presence of an aural mass with inner ear symptoms denote a sinister alarm and warrant extensive workouts. A high index of suspicion is indeed needed for early diagnosis.

The decision to repeat aural tissue biopsy is crucial in the diagnosis of temporal bone carcinoma as illustrated in this case. The initial biopsy report of infarcted polyp necessitated deeper re-biopsy and resulted in the glaring diagnosis of carcinomatous changes. Aural polyp is commonly associated with inflammatory processes, cholesteatoma, tumor as well as foreign body. The association of aural polyp with cholesteatoma is well known with incidence in children as high as $45 \% .{ }^{8}$ Meanwhile, the association between aural polyp with malignancy is rare but is more prevalent among the elderly group.

Moreover, aural polyps may represent only the tip of the iceberg and mask extensive underlying pathological processes. High resolution computed-tomography imaging is indispensable in mapping the extent of the disease and helps in prognostication. Where intracranial involvement is seen, further magnetic resonance imaging is suggested to further delineate the depth of soft tissue invasion.

More importantly, this greatly aids in staging disease as major parts of the temporal bone are not amenable to clinical assessment. This forms the basis of the T-N-M staging system proposed by Arriaga et al. ${ }^{9}$ which has been shown to provide reliable and reproducible staging segregation from a surgical point of view. T stages signify the tumor extent and pattern of infiltration as well delineate the extension of surgical resection.

With the extensive involvement of inner ear structures, the patient was classified as stage 4 temporal bone carcinoma of middle ear origin with external ear extension. There was neither presence of regional lymphadenopathy nor distant metastasis. Efforts to review the literature on optimal treatment for this tumor stage were futile due to the varied systems of tumor classification. Nonetheless, the preferred choice for temporal bone carcinoma has been radical surgery with tumor-free surgical margins. ${ }^{7,10}$ The extent of surgical resection correlates with tumor stage. Using Arriaga's classification, en-bloc excision of external ear canal for $\mathrm{T} 1$ tumors, partial temporal bone resection for $\mathrm{T} 2$ tumors and subtotal or total temporal bone resection for more advanced tumors have been recommended. ${ }^{9}$ Chee et al. ${ }^{2}$ negate routine neck dissection in patients with absence of clinical neck disease and emphasize that resection of the parotid gland deep lobe is not necessary due to the rarity of advanced tumor spread to these sites.

In cases of positive surgical margins or advanced stages, adjuvant postoperative radiotherapy and chemotherapy could improve survival. ${ }^{12}$ As in this case, sole radiotherapy is only employed whenever surgery is not feasible either in cases of intracranial extension, patient refusal or in surgically unfit patients.

In conclusion, middle ear carcinoma is rare and may masquerade as a benign aural polyp. A high index of suspicion is needed to avoid late diagnosis. Symptoms of severe otalgia and inner ear disturbances are indicators of possible malignancy, as are recent-onset symptoms of otitis media developing over a relatively short course later in life. In cases of refractory polyp where initial biopsy fails to yield positive findings, repeat deeper aural tissue biopsy is mandatory. High resolution temporal bone computed tomography is indispensable in delineating tumor extent and preoperative surgical planning as well as prognostication. Primary surgical resection of tumor with clear surgical margins, followed by postoperative radiotherapy, is the preferred treatment modality in the management of middle ear carcinoma.

\section{REFERENCES}

1. Moody SA, Hirsch BE, Myers EN. Squamous cell carcinoma of the external auditory canal: an evaluation of a staging system. Am J Otol. 2000 Jul;21(4):582-8.

2. Chee G, Mok P, Sim R. Squamous cell carcinoma of the temporal bone: diagnosis, treatment and prognosis. Singapore Med J. 2000 Sep;41(9):441-446,451.

3. Austin JR, Stewart K, Fawzi N. Squamous cell carcinoma of the external auditory canal. Therapeutic prognosis based on a proposed staging system. Arch Otolaryngol Head Neck Surg 1994 Nov;120(11):1228-32.

4. Paaske PB, Witten J, Schwer S, Hansen HS. Results in treatment of carcinoma of the external auditory canal and middle ear. Cancer 1987 Jan;59(1):156-60.

5. Noorizan Y, Asma A. Temporal bone carcinoma: a case report. Med J. Malaysia. 2010 Jun;65(2):162-164.

6. Maran AGD, Stell PM. Tumours of the skin and ear. In: Watkinson JC, Wilson JA, Gaze M, Stell PM, Maran AGD (eds). Stell and Maran's head and neck surgery. $4^{\text {th }}$ edition. Oxford: ButterworthHeinemann; 2000. 431-440.

7. Hahn SS, Kim JA, Goodchild N, Constable WC. Carcinoma of the middle ear and external auditory canal. Int J Radiat Oncol Biol Phys.1983 Jul;9(7):1003-1007.

8. Tay HL, Hussain SS. The management of aural polyps. J Laryngol Otol. 1997 Mar;111(3):212-214

9. Arriaga $M$, Curtin $H$, Takahashi H, Hirsch BE, Kamerer DB. Staging proposal for external auditory meatus carcinoma based on preoperative clinical examination and computed tomography findings. Ann Otol Rhinol Laryngol. $1990 \mathrm{Sep}$; 99(9 Pt1);714-21.

10.Pfreundner L, Schwager K, Wiillner J, Baier K, Bratengeier K, Brunner FX, Flentje M. Carcinoma of the external auditory canal and middle ear. Int. J. Radiat Oncol Biol Phys. 1999 Jul; 44(4); 777-788.

11.Graham MD, Sataloff RT, Kemink JL, Wolf GT, McGillicudy JE. Total en bloc resection of the temporal bone and carotid artery for malignant tumors of the ear and temporal bone. Laryngoscope $1984 \mathrm{Apr} ; 94(4): 528$-533.

12. Yin M, Ishikawa K, Honda K, Arakawa T, Harabuchi Y, Nagabashi T, et. al. Analysis of 95 cases of squamous cell carcinoma of the external and middle ear. Auris Nasus Larynx. 2006 Sep:33(3);251-257. 